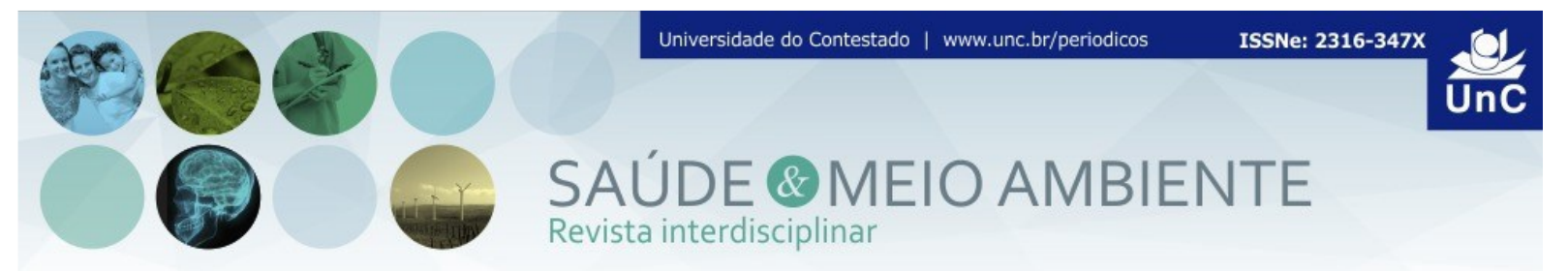

\title{
CONSUMO ALIMENTAR E SAÚDE BUCAL EM ESCOLARES DE UM MUNICÍPIO DA REGIÃO MEIO OESTE DE SANTA CATARINA
}

\section{FOOD CONSUMPTION AND ORAL HEALTH IN A MIDWEST CITY OF SANTA CATARINA}

\author{
Emanueli Lopes ${ }^{1}$ \\ Guilherme Bunn² \\ Grasieli de Oliveira Ramos ${ }^{3}$ \\ Francieli Bizzotto ${ }^{4}$ \\ Ana Paula Ariotti ${ }^{5}$ \\ Carina Rossoni ${ }^{6}$
}

\begin{abstract}
RESUMO
Introdução: A cárie dental é uma doença crônica multifatorial que apesar do seu declínio nos últimos anos, considera-se um problema de saúde pública. Objetivo: Analisar os hábitos alimentares e a relação com a saúde bucal em escolares. Metodologia: Trata-se de um estudo transversal, realizado no período de outubro a novembro de 2017, em uma cidade da Região Meio Oeste de Santa Catarina. Os dados foram obtidos através de um questionário semiestruturado e prontuário eletrônico. A análise estatística foi realizada através do GraphPad Prism $6^{\circledR}$. Resultados: A amostra constitui-se por 51 crianças, idade média 10,4 anos, prevalência do sexo feminino $(66,7 \%)$. Os maiores índices de escolares com diagnóstico de cárie dental $(25,5 \%)$ residiam na zona rural, menor renda mensal, menor escolaridade dos pais e maior frequência de consumo de alimentos cariogênicos. Conclusão: Hábitos alimentares associados às características sociodemográficas possuem relação direta refletindo na saúde bucal dos escolares, tornando-se evidente a importância da educação em saúde como medida preventiva.
\end{abstract}

Palavras chave: Educação em saúde. Alimentos cariogênicos. Alimentos cariostáticos. Cárie dental. Saúde bucal.

\footnotetext{
${ }^{1}$ Nutricionista, curso de graduação em Nutrição. Universidade do Oeste de Santa Catarina (Unoesc). Videira. Santa Catarina. Brasil. E-mail: emanueliilopes@gmail.com

${ }^{2}$ Cirurgião dentista. Secretaria de Saúde do Município de Ibicaré. Santa Catarina. Brasil. E-mail: fbunn@bol.com.br

${ }^{3}$ Cirurgiã-dentista, Curso de Graduação em odontologia e Programa de Mestrado em Biociências e Saúde. Universidade do Oeste de Santa Catarina (Unoesc). Joaçaba. Santa Catarina. Brasil. E-mail: grasieli.ramos@unoesc.edu.br

${ }^{4}$ Nutricionista, Programa de Mestrado em Biociências e Saúde. Universidade do Oeste de Santa Catarina (Unoesc). Joaçaba. Santa Catarina. Brasil. E-mail: franbizzotto@yahoo.com.br

${ }^{5}$ Nutricionista. Curso de Graduação em Nutrição. Universidade do Oeste de Santa Catarina (Unoesc). Joaçaba. Santa Catarina. Brasil. E-mail: anapaula.ariotti@unoesc.edu.br

${ }^{6}$ Nutricionista. Curso de Graduação em Nutrição e Programa de Mestrado em Biociências e Saúde. Universidade do Oeste de Santa Catarina (Unoesc). Joaçaba. Santa Catarina. Brasil. E-mail: carina.rossoni@unoesc.edu.br
} 


\begin{abstract}
Introduction: Dental caries is a chronic multifactorial disease that, despite its decline in recent years, is considered a public health problem. Objective: The aim of this study was to analyze eating habits and the relationship with oral health in schoolchildren. Methods: A cross-sectional study, carried out in the period of October, November, 2017, in a Midwest City of Santa Catarina. Data were obtained through a semistructured questionnaire and electronic medical record. A statistical analysis was performed using GraphPad Prism 6®. Results: A sample of 51 children, mean age 10.4 years, female prevalence $(66.7 \%)$; $(25.5 \%)$ residence in the rural area, lower monthly income, lower parental schooling and higher frequency of cariogenic food consumption. Conclusion: Dietary habits associated with socio-demographic characteristics are directly related to the oral health of schoolchildren, evidencing the importance of health education as a preventive measure.
\end{abstract}

Keywords: Health Education. Cariogenic foods. Cariostatic foods. Dental caries. Oral health.

\title{
INTRODUÇÃO
}

Os hábitos alimentares estão diretamente ligados a uma série de doenças crônicas, dentre elas a cárie dentária. Esta, que apesar de apresentar uma etiologia complexa, têm-se registros de que a alimentação representa um papel de destaque, tanto na saúde geral quanto na saúde bucal na infância, devido a essa ser a fase do crescimento, desenvolvimento e formação de hábitos alimentares ${ }^{1}$.

Os fatores biológicos relacionados ao processo de desenvolvimento das doenças bucais como a cárie dentária já são conhecidos, porém estão cada vez mais sendo adicionados aos determinantes desse processo fatores sociais, psicológicos e ambientais ${ }^{2}$.

Entre estas doenças bucais, podemos destacar os defeitos na estrutura dos dentes e a cárie dentária, doenças com maiores índices registrados na infância e adolescência ${ }^{3}$.

A cárie dentária é uma doença multifatorial, infecciosa, transmissível e está intimamente relacionada à introdução dos carboidratos refinados na dieta, principalmente a sacarose, portanto a alimentação pode ser vista como um fator primário de determinação da susceptibilidade para a doença. $E$ a sua prevenção deve iniciar na infância e para isso é fundamental a adoção de bons hábitos alimentares a nível familiar ${ }^{4,5,6}$.

Neste contexto, pode-se afirmar que a cárie e a obesidade têm um componente etiológico comum, à ingestão de sacarose ${ }^{7}$. Ambas são doenças multifatoriais associadas a hábitos de dieta, estilo de vida e determinantes socioculturais sugerindo uma relação entre as mesmas ${ }^{8}$. 
Desta maneira, destaca-se a importância de se estabelecer um padrão alimentar adequado para criança, pois será uma herança carregada para fase adulta. A implantação de um padrão alimentar saudável contribui não só para o desenvolvimento satisfatório da criança, como também auxilia na prevenção de doenças crônicas, como a cárie dentária ${ }^{9}$.

Sendo assim, este estudo teve como objetivo avaliar os hábitos alimentares e a sua relação com a saúde bucal de escolares no Município de Ibicaré, Região Meio Oeste de Santa Catarina, Brasil.

\section{METODOLOGIA}

Este estudo é de natureza aplicada, transversal, de cunho quantitativo, exploratória descritiva, realizado com 51 estudantes da rede municipal de ensino do Município de Ibicaré, SC, no período de outubro a novembro de 2017. Foram incluídos no estudo escolares da rede municipal de ensino que realizaram avaliação odontológica na Unidade Básica de Saúde de Ibicaré no segundo semestre de 2017, foram excluídos do estudo os escolares que não tiveram o Termo de Consentimento Livre e Esclarecido Participação de Menor autorizado pelo responsável, os que não possuíam registro clínico em seu prontuário ou ainda os que não preencheram o questionário completamente. O projeto de pesquisa foi aprovado pelo Comitê de Ética em Pesquisa, sob parecer $n^{\circ} 2.237 .667$.

As variáveis foram obtidas através de:

2.1 Características sociodemográficas e clínicas: foram obtidas através de um questionário elaborado pelos pesquisadores, semiestruturado abrangendo os com itens: localização da moradia se é própria, alugada ou cedida, quantas pessoas residem na casa, nível de escolaridade dos pais e renda familiar mensal, dentre outros. As informações clínicas foram obtidas através da consulta ao prontuário eletrônico do paciente, disponível na Secretaria Municipal de Saúde de Ibicaré.

2.2 Hábitos alimentares: obtidos através de um Questionário de Frequência de Consumo Alimentar (QFCA), adaptado de acordo com Godoy (2013) ${ }^{9}$ constituído por alimentos com características cariogênicas e cariostáticas. Além deste, questões que abordavam o local e frequência das refeições realizadas pelos escolares

2.3 Saúde bucal: foram obtidos através da avaliação odontológica registrada em prontuário eletrônico disponível na Secretária Municipal de Saúde do Município de Ibicaré, onde consta se há ou não presença de cárie dental no paciente avaliado.

Os resultados são apresentados como média \pm desvio padrão. Os dados foram analisados utilizando-se o Teste $t$ de Student para amostras pareadas (pré e pósintervenção) e para amostras independentes (entre os gêneros). Diferenças entre os grupos foram consideradas significativas quando $p \leq 0,05$. Todas as análises foram realizadas com o programa GraphPad Prism $6^{\circledR}$. 


\section{RESULTADOS}

51 escolares da rede pública de ensino, matriculados na $4^{\mathrm{a}}$ e $5^{\mathrm{a}}$ série do ensino fundamental constituíram a amostra deste estudo. Houve a prevalência na participação de meninas $(66,7 \%)$ com média de idade 10,4 anos dos participantes.

A presença de cárie dental esteve presente em $25,5 \%$ dos escolares avaliados, sendo que mais da metade $(53,8 \%)$ foram diagnosticados nas meninas. Ao analisar os dados socioeconômicos, constatou-se que $61,5 \%$ dos escolares com diagnóstico de cárie dental residiam na zona rural do município e com renda mensal de até um salário mínimo (46,1\%). Nota-se ainda a relação da baixa escolaridade dos pais com maior índice de cárie dental nos filhos $(p=0,7490)$ (Tabela 1).

Tabela 1 - Características sociodemográficas dos escolares do Município de Ibicaré, Região Meio Oeste de Santa Catarina, Brasil.

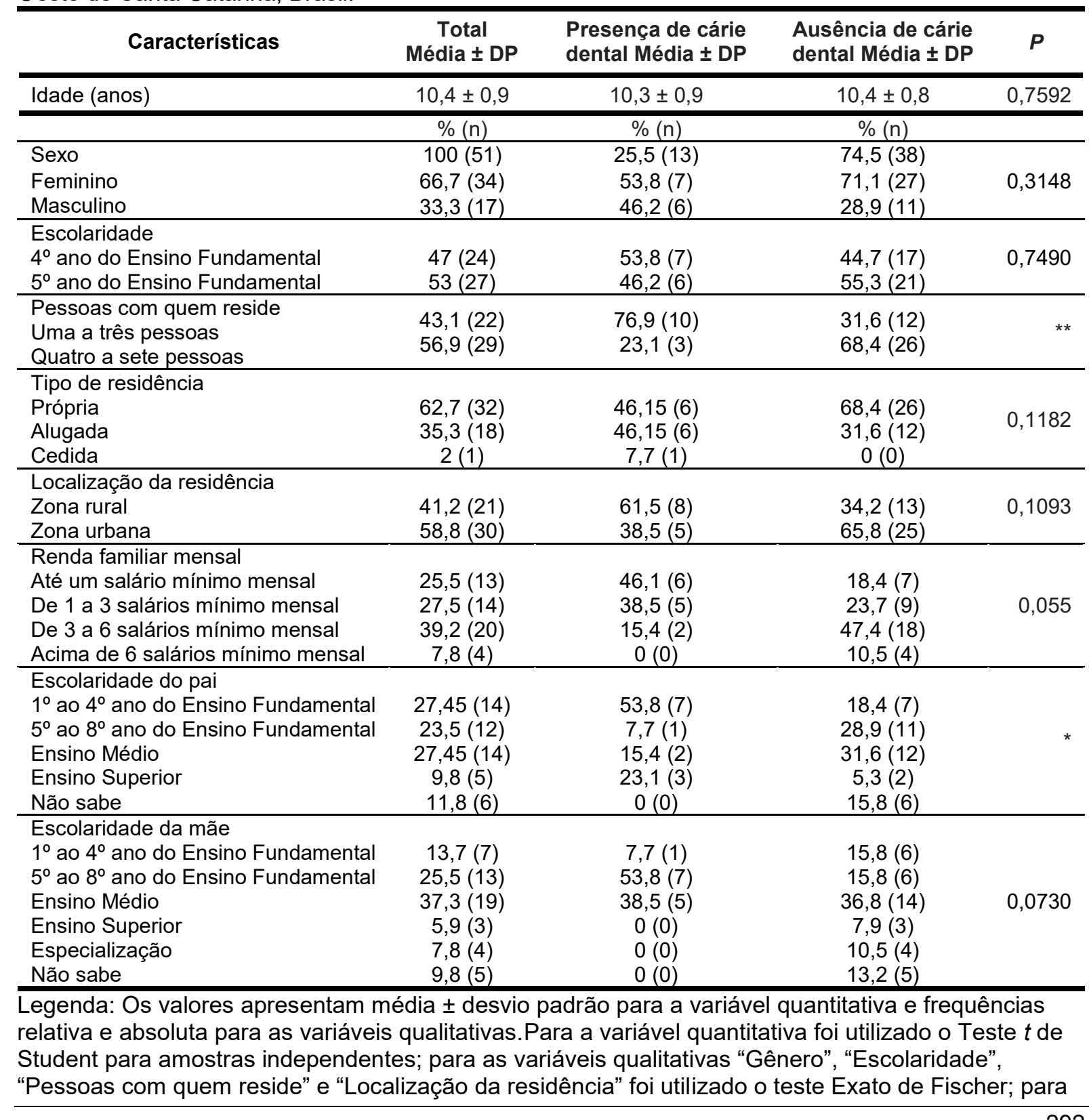


as variáveis qualitativas "Tipo de residência", "Renda familiar mensal", "Escolaridade do pai" e "Escolaridade da mãe", foi utilizado o teste do Qui-quadrado de Pearson. A diferença estatística foi considerada significativa quando $P<0,05$. Fonte: Os autores.

Tabela 2 - Frequência e local das refeições realizadas pelos escolares do Município de Ibicaré, Região Meio Oeste de Santa Catarina, Brasil.

\begin{tabular}{|c|c|c|c|c|}
\hline $\begin{array}{l}\text { Frequência das } \\
\text { refeições e local }\end{array}$ & $\begin{array}{c}\text { Total } \\
\%(n) \\
100(51)\end{array}$ & $\begin{array}{c}\text { Presença de cárie dental } \\
\%(n) \\
25,5(13)\end{array}$ & $\begin{array}{c}\text { Ausência de cárie dental } \\
\%(n) \\
74,5(38)\end{array}$ & $P$ \\
\hline $\begin{array}{l}\text { Café da manhã } \\
\text { Nenhuma } \\
\text { Até duas } \\
\text { vezes/semana } \\
\text { Todos os dias } \\
\end{array}$ & $\begin{array}{c}13,7(7) \\
2(1) \\
84,3(43)\end{array}$ & $\begin{array}{c}15,4(2) \\
7,7(1) \\
76,9(10)\end{array}$ & $\begin{array}{c}13,2(5) \\
0(0) \\
86,2(33)\end{array}$ & 0,2153 \\
\hline $\begin{array}{l}\text { Local } \\
\text { Em casa } \\
\text { Não faz }\end{array}$ & $\begin{array}{c}92,2(47) \\
7,8(4)\end{array}$ & $\begin{array}{c}84,6(11) \\
15,4(2)\end{array}$ & $\begin{array}{c}94,7(36) \\
5,3(2)\end{array}$ & 0,2658 \\
\hline $\begin{array}{l}\text { Lanche da manhã } \\
\text { Nenhuma } \\
\text { Até } 2 x / \text { semana } \\
\text { De } 3 x \text { a } 6 x / \text { semana } \\
\text { Todos os dias }\end{array}$ & $\begin{array}{c}41,2(21) \\
11,8(6) \\
29,4(15) \\
17,6(9) \\
\end{array}$ & $\begin{array}{l}46,1(6) \\
15,4(2) \\
23,1(3) \\
15,4(2)\end{array}$ & $\begin{array}{c}39,5(15) \\
10,5(4) \\
31,6(12) \\
18,4(7) \\
\end{array}$ & 0,8992 \\
\hline $\begin{array}{l}\text { Local } \\
\text { Em casa } \\
\text { Na escola } \\
\text { Não faz }\end{array}$ & $\begin{array}{l}37,2(19) \\
21,6(11) \\
41,2(21)\end{array}$ & $\begin{array}{l}30,8(4) \\
15,4(2) \\
53,8(7)\end{array}$ & $\begin{array}{c}39,5(15) \\
23,7(9) \\
36,8(14)\end{array}$ & 0,5525 \\
\hline $\begin{array}{l}\text { Almoço } \\
\text { Nenhuma } \\
\text { De 3x a 6x/semana } \\
\text { Todos os dias } \\
\end{array}$ & $\begin{array}{c}3,9(2) \\
2(1) \\
94,1(48) \\
\end{array}$ & $\begin{array}{c}0(0) \\
7,7(1) \\
92,3(12) \\
\end{array}$ & $\begin{array}{c}5,3(2) \\
0(0) \\
94,7(36) \\
\end{array}$ & 0,1642 \\
\hline $\begin{array}{l}\text { Local } \\
\text { Em casa }\end{array}$ & $100(51)$ & 100 (13) & $100(38)$ & \\
\hline $\begin{array}{l}\text { Lanche da tarde } \\
\text { Nenhuma } \\
\text { Até } 2 x / \text { semana } \\
\text { De } 3 x \text { a } 6 x / \text { semana } \\
\text { Todos os dias }\end{array}$ & $\begin{array}{c}9,8(5) \\
11,8(6) \\
15,7(8) \\
62,7(32)\end{array}$ & $\begin{array}{l}32,1(3) \\
15,4(2) \\
0(0) \\
61,5(8)\end{array}$ & $\begin{array}{c}5,3(2) \\
10,5(4) \\
21(8) \\
63,2(24)\end{array}$ & 0,1082 \\
\hline $\begin{array}{l}\text { Local } \\
\text { Em casa } \\
\text { Na escola } \\
\text { Não faz } \\
\end{array}$ & $\begin{array}{c}21,6(11) \\
72,5(37) \\
5,9(3) \\
\end{array}$ & $\begin{array}{l}15,4(2) \\
61,5(8) \\
32,1(3)\end{array}$ & $\begin{array}{c}23,7(9) \\
76,3(29) \\
0(0) \\
\end{array}$ & ** \\
\hline $\begin{array}{l}\text { Jantar } \\
\text { Nenhuma } \\
\text { De } 3 x \text { a } 6 x / \text { semana } \\
\text { Todos os dias } \\
\end{array}$ & $\begin{array}{r}3,9(2) \\
7,9(4) \\
88,2(45) \\
\end{array}$ & $\begin{array}{c}0(0) \\
0(0) \\
100(13) \\
\end{array}$ & $\begin{array}{c}5,3(2) \\
10,5(4) \\
84,2(32) \\
\end{array}$ & 0,3125 \\
\hline $\begin{array}{l}\text { Local } \\
\text { Em casa } \\
\text { Não faz }\end{array}$ & $\begin{array}{c}98(50) \\
2(1)\end{array}$ & $\begin{array}{c}92,3(12) \\
7,7(1)\end{array}$ & $\begin{array}{c}100(38) \\
0(0)\end{array}$ & 0,2549 \\
\hline $\begin{array}{l}\text { Lanche da noite } \\
\text { Nenhuma } \\
\text { Até } 2 x / \text { semana } \\
\text { De } 3 x \text { a } 6 x / \text { semana } \\
\text { Todos os dias }\end{array}$ & $\begin{array}{c}60,8(31) \\
17,6(9) \\
13,7(7) \\
7,9(4) \\
\end{array}$ & $\begin{array}{c}69,2(9) \\
23,1(3) \\
0(0) \\
7,7(1) \\
\end{array}$ & $\begin{array}{c}57,9(22) \\
15,8(6) \\
18,4(7) \\
7,9(3) \\
\end{array}$ & 0,4087 \\
\hline $\begin{array}{l}\text { Local } \\
\text { Em casa } \\
\text { Não faz }\end{array}$ & $\begin{array}{l}47,1(24) \\
52,9(27)\end{array}$ & $\begin{array}{l}30,8(4) \\
69,2(9)\end{array}$ & $\begin{array}{l}52,6(20) \\
47,4(18)\end{array}$ & 0,2111 \\
\hline
\end{tabular}

Legenda: Os valores apresentam as frequências relativa e absoluta para as variáveis qualitativas. Para as variáveis qualitativas "Local do café da manhã", "Local do jantar" e "Local do lanche da noite" foi utilizado o teste Exato de Fischer; para as variáveis qualitativas "Café da manhã", "Lanche da manhã", "Local lanche da manhã", "Almoço", "Lanche da tarde", "Local lanche da tarde", "Jantar" e 
"Lanche da noite", foi utilizado o teste do Qui-quadrado de Pearson. A diferença estatística foi considerada significativa quando $P<0,05$. Fonte: Os autores.

Com relação à alimentação, evidencia-se que as refeições são realizadas no ambiente familiar ou escola. Na escola, apenas uma refeição é servida: lanche, de acordo com os dados apresentados na tabela 2. Evidencia-se o maior consumo de alimentos cariogênicos em portadores de cárie dental, como bolos, biscoitos recheados, refrigerantes e açúcares (figura 1A). E o consumo de alimentos cariostáticos, carnes, leites, legumes e frutas, observa-se maior frequência de consumo em crianças não portadoras de cárie dental, evidenciando assim seus benefícios a saúde (figura 1B).

Figura 1 - Frequência de consumo de alimentos cariogênicos e cariostáticos dos escolares do Município de Ibicaré SC, 2017.

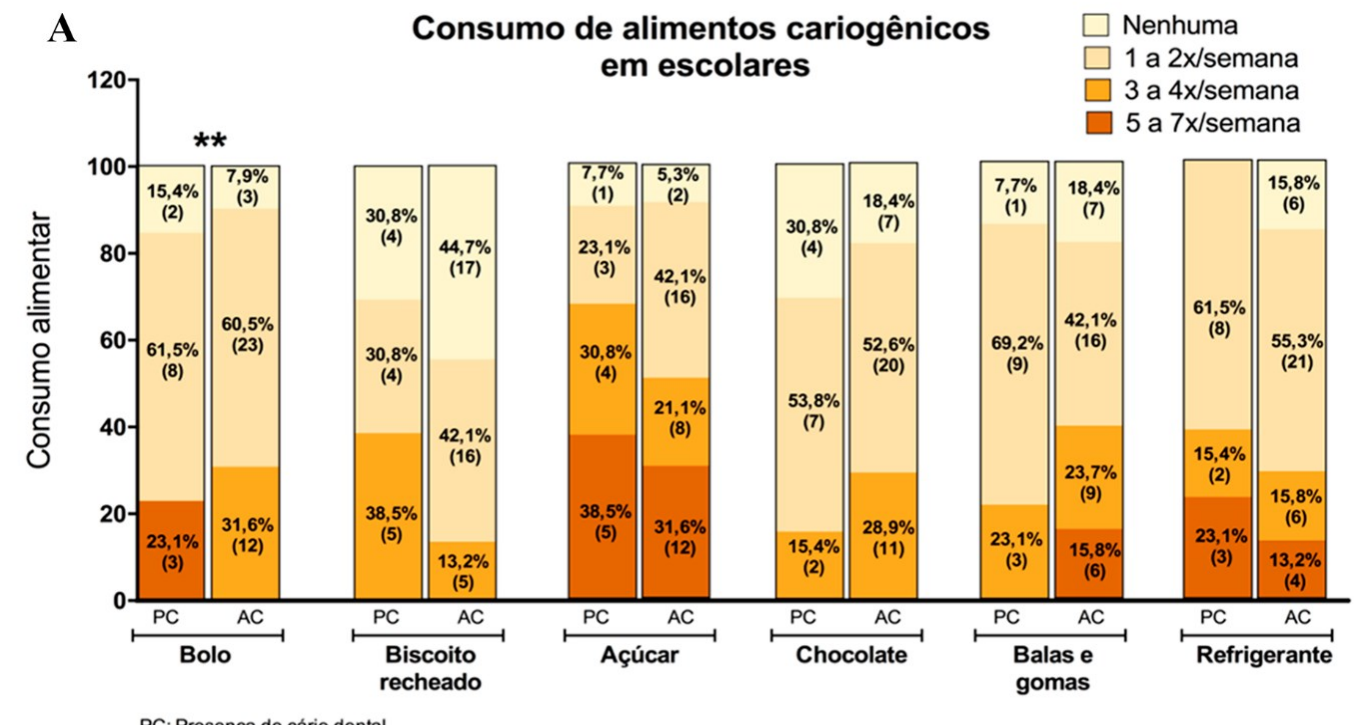

PC: Presença de cárie dental AC: Ausência de cárie dental

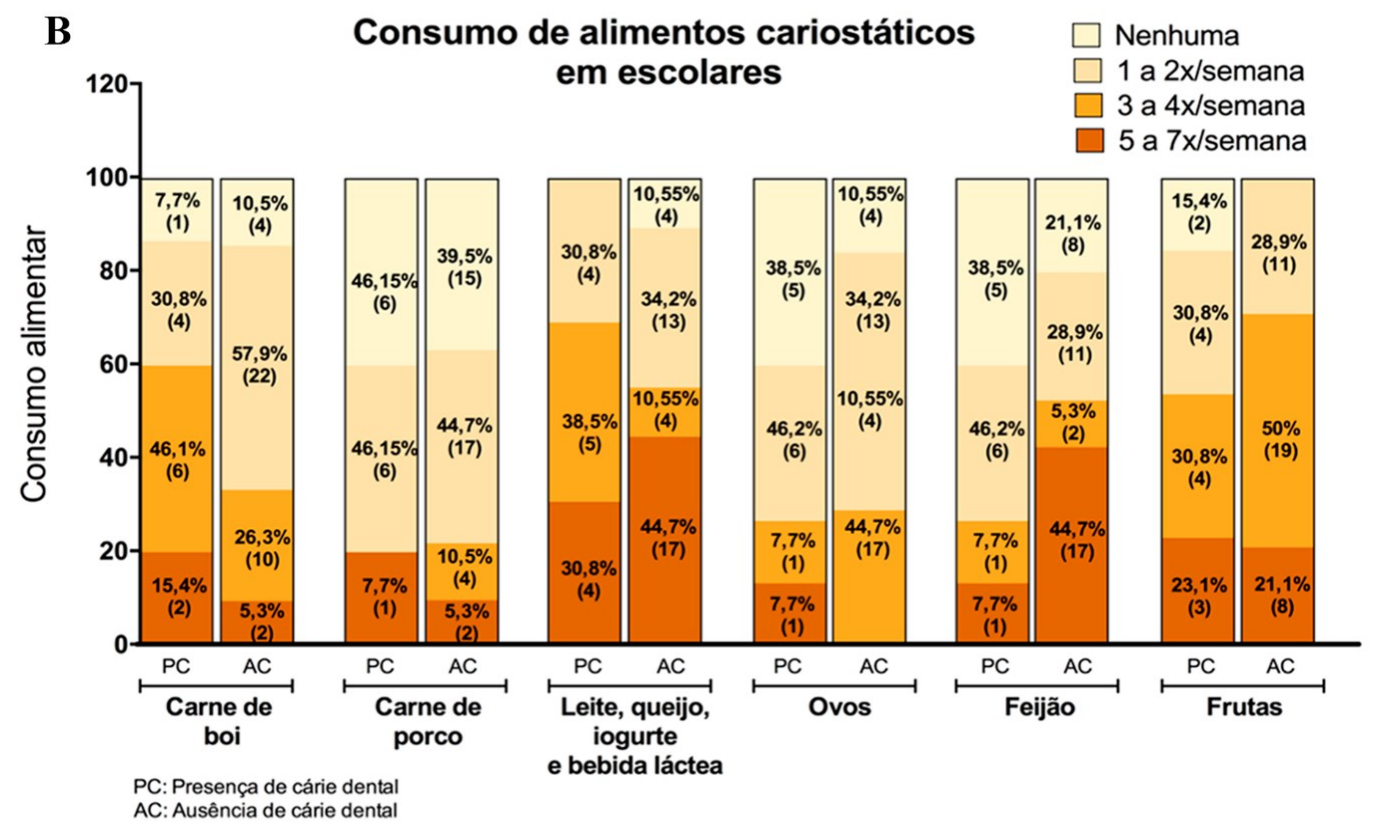

Legenda: $\mathrm{PC}=$ presença de cárie dentária; $\mathrm{AC}$ = ausência de cárie dentária 


\section{DISCUSSÃO}

A alimentação na infância é marcada por um comportamento instável: o volume de alimentos consumido pode ser grande em alguns momentos e mínimo em outros. Algumas teimosias podem fazer com que o alimento preferido de hoje seja intolerável outro dia, ou que somente um alimento seja aceito por vários dias seguidos. Se os pais não julgarem essa atitude como transitória e agirem com medidas arbitrárias, isso poderá se tornar um distúrbio alimentar real e permanecer em fases posteriores. $\mathrm{O}$ pré-escolar tem como características a dificuldade em aceitar alimentos novos e a recusa por uma ampla diversidade de alimentos ${ }^{10}$.

Muitas influências, umas evidentes e outras nem tanto, estabelecem o consumo alimentar e os hábitos das crianças. Os costumes, preferências e aversões são determinados nos primeiros anos e carregados até a idade adulta. As influências mais relevantes referentes ao consumo alimentar na fase de desenvolvimento abrangem os interesses sociais, o ambiente familiar, os meios de comunicação, a influência dos colegas e doenças ${ }^{11}$.

A prática alimentar é um significativo elemento etiológico comum da obesidade, as doenças dela decorrentes e a cárie dentária ${ }^{12}$. A alimentação adequada, desempenha uma função importante na determinação da saúde bucal, e consegue, dessa forma, ajudar na prevenção da saúde da pessoa no decorrer da vida. Programas de intervenção educacional destinados a população são importantes para fomentar à adoção de hábitos e práticas alimentares favoráveis e, assim, um estado nutricional adequado ${ }^{13}$.

Constata-se a importante relação da alimentação e a saúde bucal, neste estudo com escolares do município de Ibicaré, município da Região Meio Oeste de Santa Catarina. Os principais achados demonstram que as crianças com diagnóstico de cárie dental são as residentes na zona rural, que apresentam menor renda mensal, menor escolaridade dos pais e maior frequência de consumo de alimentos cariogênicos.

A participação de meninas prevaleceu no nosso estudo, dados semelhantes aos obtidos por Lagreca (2007) ${ }^{14}$ que avaliou os fatores de riscos associados à doença cárie dentária incidentes sobre a população de escolares em Xerém, Duque de Caxias no Estado do Rio de Janeiro. A sua amostra constitui-se por $55,2 \%$ de participantes do sexo feminino, $65,1 \%$ das crianças avaliadas apresentavam cárie dentária, dados divergentes dos apresentados em nosso estudo onde constatamos a presença de cárie dental em $25,5 \%$ dos escolares.

Ao analisar as condições socioeconômicas, os resultados foram semelhantes aos apresentados por Crispim e Cols $(2007)^{6}$, no qual a prevalência da cárie acometeu em maior porcentagem em escolares com classificação socioeconômica $C$ ou $D$, $49,2 \%$ e $38 \%$ respectivamente.

Quanto à escolaridade dos pais, Maltz e Silva $(2001)^{15}$ estabeleceram a associação entre os escolares com mais de quatro superfícies comprometidas e pais 
que apresentavam escolaridade primeiro grau incompleto. Os escolares livres de cárie foram localmente associados a pais de nível superior completo. Bem como os apresentados por Peres et al. $(2003)^{16}$, onde a renda familiar, classe social, escolaridade do pai e da mãe foram fortemente associadas à ocorrência de cárie.

No que se diz respeito à localização da residência, os resultados obtidos no nosso estudo são semelhantes aos obtidos por outros autores ${ }^{17}$, 18. Estes evidenciaram que a prevalência de escolares livres de cárie foi maior na área urbana, demonstrando que estes possuem melhores indicativos de saúde bucal que os residentes na zona rural.

Gimenez e Pontes (2011) $)^{19}$ analisaram a prevalência de carie dentária em escolares de um município da zona rural e constataram relação destes com o baixo poder aquisitivo da população. Pode-se afirmar ainda, que há dificuldade ao acesso a produtos de higiene e aos serviços de saúde.

Além disso, Silva (2007) ${ }^{20}$ observou uma forte relação entre o consumo de alimentos ricos em açúcar e a cárie dentária, mesmo considerando outros fatores etiológicos da doença bucal. Resultados semelhantes foram publicados por Liena \& Forner $(2008)^{21}$, o consumo de alimentos cariostáticos foi prevalente nos escolares com idade entre 6 a 10 anos, na Espanha, que não apresentaram cárie dental. 0 consumo de elevado de frutas durante a semana foi associado com menores índices desta doença bucal.

Apesar do Projeto Saúde Bucal Brasil, 2010 revelar uma importante redução no número de casos de cárie dentária no país, o mesmo destaca ressalta a importância da atenção a alguns aspectos, tais como a necessidade de políticas voltadas à prevenção, num modelo de atenção direcionada a Vigilância à Saúde ${ }^{22}$.

O presente estudo apresenta limitações relacionadas ao tamanho da amostra e ao período de realização, somente com alunos com diagnóstico odontológico do segundo semestre de 2017. Entretanto os resultados obtidos refletem a realidade local, o que permite a realização das intervenções em saúde de forma individualizada.

\section{CONCLUSÕES}

Conclui-se que o consumo alimentar e as características sociodemográficas tiveram relação direta na saúde bucal dos escolares avaliados no presente estudo.

Sendo assim, torna-se evidente a importância da educação em saúde como medida preventiva, através de atividades voltadas ao âmbito da nutrição e odontologia, as quais interligadas permitirão a adoção de bons hábitos de higiene e alimentares, melhorando a qualidade da saúde bucal. 


\section{REFERÊNCIAS}

1 - Morikava SM. O que é mais importante para a prevenção de cárie em crianças: restringir o contato com alimentos cariogênicos ou estimular uma alimentação saudável?. Curitiba. Dissertação [Mestrado em Odontologia] - Programa de PósGraduação em Odontologia, Departamento de Estomatologia, Setor de Ciências de Saúde, Universidade Federal do Paraná; 2015.

2 - Figueiredo MC, Ribeiro CBS, Noronha MS, Silva, KVCL. Descrição do padrão alimentar e da saúde bucal de crianças do sul do Brasil. RFO 2014 dez;19(3):329-336.

3 - Silva CM, Basso DF, Locks A. Alimentação na primeira infância: abordagem para a promoção da saúde bucal. Rev Sul-Bras Odontol 2010 jan;7(4):458-65.

4- Dias ACG, Raslan S, Scherma AP. Aspectos nutricionais relacionados à prevenção de cáries na infância. ClipeOdonto - UNITAU 2011;3(1):37-44.

5 - Peres MA, et al. Oral diseases: a global public health challenge. The Lancet 2019;394:249-260.

6 - Crispim MGA, Grillo LP, Próspero ELS, Mariath AB. Saúde bucal e sua associação com o estado nutricional e a condição socioeconômica em adolescentes. RGO 2010 jan/mar;58(1):41-46.

7 - Barbosa ML. Relação entre obesidade e cárie dental: revisão de literatura. Piracicaba. Monografia [Especialização em saúde coletiva e da família] Faculdade de Odontologia, Universidade Estadual de Campinas; 2015.

8 - Gil GS. Marcador de aceitação alimentar para cárie dentária em pré-escolares. Curitiba. Dissertação [Mestrado em Odontologia] - Programa de Pós-graduação em Odontologia, Setor de Ciências da Saúde, Universidade Federal do Paraná; 2015.

9 - Godoy SS de. Estudo da satisfação da alimentação escolar. Medianeira. Trabalho de Conclusão de Curso [Especialização] - Universidade Tecnológica Federal do Paraná; 2013.

10 - Weffort, VRS et al. Alimentação do lactente à adolescência. In: Burns DAR, et al. (Organizadores). Tratado de Pediatria 2. 4. ed. Barueri: Manole; 2017. p. 1407-1419.

11- Ogata B, Feucht SA, Lucas BL. Nutrição na Infância. In: Mahan LK, Raymond JLK. Alimentos, Nutrição e Dietoterapia. 14. ed. Rio de Janeiro: Elsevier; 2018. p. 314-330. 
12 - Traebert J. et al. Transição alimentar: problema comum à obesidade e à cárie dentária. Revista de Nutrição. 2004 abr/jun;17(2):247-253. Acesso em 02 jul. 2018. Disponível em: http://www.scielo.br/pdf/rn/v17n2/21137.pdf.

13 - Batista LRV, Moreira EAM, Corso ACT. Alimentação, estado nutricional e condição bucal da criança. Revista de Nutrição. 2007 mar/abr;20(2):191-196. Acesso em 30 de maio de 2018. Disponível em: http://www.scielo.br/scielo.php?script=sci arttext\&pid=S1415$\underline{52732007000200008 .}$.

14 - Lagreca BT. Avaliação dos fatores de risco associados à doença cárie dentária que incidem sobre a população de escolares em Xerém - Duque de Caxias. Duque de Caxias. Dissertação [Mestrado em Odontologia] - Programa de PósGraduação em Odontologia, Universidade do Grande Rio; 2007.

15 - Maltz M, Silva BB. Relação entre cárie, gengivite e fluorose e nível socioeconômico em escolares. Rev Saúde Pública. 2001;35(2):170-176.

16 - Peres MA, et al. Determinantes sociais e biológicos da cárie dentária em crianças de 6 anos de idade: um estudo transversal aninhado numa coorte de nascidos vivos no Sul do Brasil. Rev. Bras. Epidemiol. 2003; 6(4):293-306.

17 - Mello TRC, Antunes JLF. Prevalência de cárie dentária em escolares da região rural de Itapetininga, São Paulo, Brasil. Cad. Saúde Pública. 2004 maio/jun;20(3)829-835.

18 - Campos Mello TR, Antunes JLF, Waldman EA. Prevalência de cárie não tratada na dentição decídua em áreas urbanas e rurais do Estado de São Paulo, Brasil. Rev Panam Salud Publica. 2008;23(2)78-84.

19 - Gimenez ACR, Pontes ERJC. Prevalência de carie dentaria e condições periodontais de escolares de 5 a 12 anos de idade, em um município rural brasileiro. RGO - Rev Gaucha Odontol. 2011 out/dez;59(4)577-582.

20 - Silva JMF. Avaliação da frequência do consumo de alimentos cariogênicos e sua relação com a cárie dentária numa amostra de crianças. Porto. [Trabalho de conclusão de curso] - Faculdade de ciências da nutrição e alimentação, Universidade do Porto; 2007.

21 - Liena C, Forner L. Dietary habits in a child population in relation to caries experience. Caries Res. 2008;42(5)387-93.

22 - Roncalli AG. Projeto Saúde Bucal 2010. Cad. Saúde Pública. 2011 jan;27(1)4-5.

Artigo recebido em: 04/06/2020

Artigo aprovado em: 27/10/2020

Artigo publicado em: 02/12/2020 